\title{
A Fast SSVEP-Based Brain-Computer Interface ${ }^{\star}$
}

\author{
Tania Jorajuría ${ }^{[0000-0002-6493-7770]}$, Marisol Gómez ${ }^{[0000-0003-3431-1256]}$, and \\ Carmen Vidaurre(*)[0000-0003-3740-049X] \\ Department of Statistics, Informatics and Mathematics \\ Universidad Pública de Navarra, Pamplona, Spain \\ (*) carmen dot vidaurre at unavarra dot es
}

\begin{abstract}
Literature of brain-computer interfacing (BCI) for steadystate visual evoked potentials (SSVEP) shows that canonical correlation analysis (CCA) is the most used method to extract features. However, it is known that CCA tends to rapidly overfit, leading to a decrease in performance. Furthermore, CCA uses information of just one class, thus neglecting possible overlaps between different classes. In this paper we propose a new pipeline for SSVEP-based BCIs, called corrLDA, that calculates correlation values between SSVEP signals and sine-cosine reference templates. These features are then reduced with a supervised method called shrinkage linear discriminant analysis that, unlike CCA, can deal with shorter time windows and includes between-class information. To compare these two techniques, we analysed an open access SSVEP dataset from 24 subjects where four stimuli were used in offline and online tasks. The online task was performed both in control condition and under different perturbations: listening, speaking and thinking. Results showed that corrLDA pipeline outperforms CCA in short trial lengths, as well as in the four additional noisy conditions.
\end{abstract}

Keywords: Brain-computer interface - Steady-state visual evoked potential - Linear discriminant analysis - Canonical correlation analysis.

\section{Introduction}

Brain-computer interfaces (BCI) use brain signals, and most commonly electroencephalogram (EEG) to establish a new way to interact with our environment directly with mental activity. Their most common applications include the control of external devices $[10,19]$, communication tools for disabled people $[26,41]$ and neurological rehabilitation $[6,7]$. However, they can also be applied to other fields such as entertainment $[1,23]$ and marketing [32].

There are several types of brain responses that can be used to achieve these goals, such as event-related potentials [13,30], sensorimotor rhythms [12,14,22, $25,28,33-36,38]$ and visual-evoked potentials (VEP) $[18,39,40]$. One type of VEP is the steady-state visual evoked potential (SSVEP) which, in comparison to other types of evoked potentials, is a robust phenomenon [9] with high signal-tonoise ratio [31]. In particular, SSVEPs are natural responses to visual stimulation

* This research was supported by MINECO (RYC-2014-15671). 
at specific frequencies, where the brain generates oscillatory responses at the same (or multiples of) frequency of the visual stimulus at which the subject focuses.

Over the past years, different methodologies have been followed to analyse SSVEP signals. Among them, the most widely used method is the canonical correlation analysis (CCA) $[2,5,17]$. CCA has also been extended $[44,45]$ and combined [8] with other methods. The concept underlying CCA-based methods is the maximization of the correlation between SSVEP signals and sine-cosine reference matrices and typically, they do not need any calibration data. This aspect offers an advantage in comparison to other methods, because the BCI system is immediately ready-to-use by a new participant. Nevertheless, CCA maximizes the correlation of multivariate datasets without minimizing the overlap between different classes, thus leading to poor performance, for example, when trials are not sufficiently long or the number of dimensions (channels) is large.

In this paper we propose a new pipeline to extract and classify SSVEP features, based on linear discriminant analysis (LDA) $[24,27,43]$ with shrinkage $[3,37]$. The linear discriminant aims to minimize the within-class covariance at the same time that maximizes the between-class covariance. Thus, it is a supervised method that finds a linear combination of the input features that best separates them according to its objective function. Our results show that the proposed SSVEP-based BCI system outperforms the state-of-the-art CCA method when trials are as short as 1 second. Besides, in out-of-the-lab conditions and without prior knowledge of perturbations structure, its performance is also superior to CCA. This new pipeline is also practical, since there is no need to select subject-specific hyper-parameters.

\section{Materials}

We analysed open access data described and shared by [11]. In that study, data of 24 subjects were analysed after acquiring written informed consent by participants. In this paper, we used the same datasets for our analyses.

EEG from 60 channels were recorded with $1 \mathrm{kHz}$ sampling rate, setting left mastoid as the reference electrode. Stimuli markers that specified the start and end of the flickering were used to segment EEG.

Each participant was presented a screen with four circles placed at different locations with different flickering frequencies: $5.45 \mathrm{~Hz}$ (up), $8.57 \mathrm{~Hz}$ (down), 12 $\mathrm{Hz}$ (right) and $15 \mathrm{~Hz}$ (left). In order to prevent that these main frequencies coincide with their higher harmonics, frequencies multiples of each other were avoided. Offline and online tasks were carried out by all subjects. In this paper, data acquired from offline task were used as the training dataset, whereas online task data were used as the testing dataset.

In each trial during the offline session, the participants were asked to focus on one of the four randomly presented flickering circles for three seconds. After 
the stimuli, they could blink during 1 second. In total, each subject performed 25 trials for each of the four stimuli.

On the other hand, during the online task the subjects freely selected one of the four circles and focused on it for three seconds. Then, they were presented the classification result and they had to either confirm or reject this result. If the result was rejected, the subjects had to specify the correct circle using the keyboard. Furthermore, the online task was performed under four different environmental conditions: control (i.e. no perturbation), listening, speaking and thinking. In total, each subject performed 100 trials for each condition. For more details on data acquisition procedure, please refer to [11].

\section{Methods}

In this manuscript we compare two pipelines to extract features for an SSVEPbased BCI system: the state-of-the-art CCA method and the proposed approach, that we named corrLDA. The notation used in this paper is boldface capital letters to denote matrices (e.g. $\mathbf{X}$ ), boldface lower-case letters for vectors (e.g. $\mathbf{x}$ ) and regular letters for scalars (e.g. $x$ ).

\subsection{Pre-processing}

In the two analysed pipelines, the EEG data was de-trended and band-pass filtered between 0.53 and $40 \mathrm{~Hz}$ with a fourth order Butterworth filter to remove $\mathrm{DC}, 50 \mathrm{~Hz}$ power line noise and high frequency artifacts.

\subsection{Canonical Correlation Analysis (CCA)}

The standard CCA method was selected as baseline, because it is considered the most popular technique to extract features from SSVEP signals $[2,5,9,17$, 21,42]. Briefly, CCA aims at finding the maximal correlation between the EEG signals and several reference signals (one or more at different frequencies) to estimate the similarity between the EEG and each of the flickering frequencies (classes); i.e., CCA aims at finding the SSVEP. In [11] canonical correlations were calculated between the EEG and two sine-cosine reference signal matrices. The reference functions of one of these two matrices were defined at the frequencies corresponding to the stimulation, whereas the frequencies of the other matrix corresponded to their second harmonics. Therefore, two canonical correlation values were obtained for each of the eight reference signals, which were used as features for classification. In order to obtain results for this study, we proceeded similarly.

\subsection{CorrLDA}

The proposed corrLDA pipeline (see Fig. 1) consists of correlating each trial in EEG pre-processed data with a sine-cosine reference matrix. This matrix is 
composed of $N=16$ signals that match with sines and cosines of the four flickering frequencies and their corresponding second harmonics. This way, the dimensions of resulting data are $N$ by number of channels by number of trials.

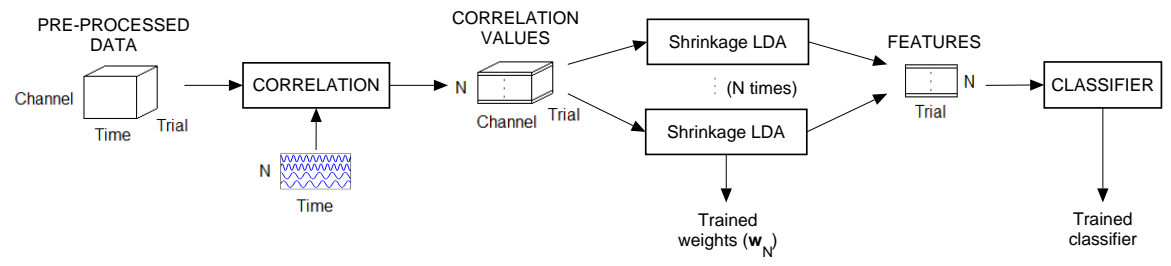

Fig. 1. corrLDA block scheme $(N=16)$.

Then, these correlation values are projected using regularized linear discriminant analysis with shrinkage (shrinkage LDA), implemented in the BBCI Toolbox of Matlab [4]. The LDA was regularized because of the high-dimensionality of the features (60) in comparison to the number of trials per class (25). Recall that the linear discriminant analysis (LDA) finds a one-dimensional subspace in which the classes are well (linearly) separated. This is formalized by requiring that after the projection onto the subspace, the ratio of the between-class variance to the within-class variance is maximal:

$$
\mathbf{w}=\underset{\mathbf{w}}{\operatorname{argmax}} \frac{\left\{\mathbf{w}^{T} \mathbf{S}_{b} \mathbf{w}\right\}}{\left\{\mathbf{w}^{T} \mathbf{\Sigma} \mathbf{w}\right\}}
$$

where $\mathbf{S}_{b}$ and $\boldsymbol{\Sigma}$ are between-class and within-class scatter matrices, respectively, computed as:

$$
\begin{gathered}
\mathbf{S}_{b}=\left(\boldsymbol{\mu}_{1}-\boldsymbol{\mu}_{2}\right)\left(\boldsymbol{\mu}_{1}-\boldsymbol{\mu}_{2}\right)^{T} \\
\boldsymbol{\Sigma}=\frac{\boldsymbol{\Sigma}_{1}+\boldsymbol{\Sigma}_{2}}{2}
\end{gathered}
$$

where $\boldsymbol{\mu}_{1}$ and $\boldsymbol{\mu}_{2}$ are the sample class means and $\boldsymbol{\Sigma}_{1}$ and $\boldsymbol{\Sigma}_{2}$ are the sample covariances for class 1 and 2 respectively. The optimization problem in Eq. 1 amounts to solving a generalized eigenvalue decomposition (GEVD), which solves $\mathbf{S}_{b} \mathbf{w}=\beta \mathbf{\Sigma} \mathbf{w}$ for the leading eigenvector. Thus, the optimal subspace is defined by:

$$
\mathbf{w}=\boldsymbol{\Sigma}^{-1}\left(\boldsymbol{\mu}_{1}-\boldsymbol{\mu}_{2}\right)
$$

As the covariance matrix is often typically poorly conditioned, we follow the approach by Ledoit and Wolf $[15,16]$ and replace in Eq. 4 by a shrinkage estimate of the form:

$$
\boldsymbol{\Sigma}_{\lambda}=(1-\lambda) \tilde{\boldsymbol{\Sigma}}+\lambda \tilde{\boldsymbol{\Sigma}}, \lambda \in[0,1]
$$

The matrix $\tilde{\boldsymbol{\Sigma}}$ is the sample-covariance matrix of a restricted sub-model, and the optimal shrinkage intensity $\lambda$ can be estimated from the data. We use the 
following sub-model: all variances (i.e. all diagonal elements) are equal, and all covariances (i.e. all off-diagonal elements) are zero (see [29] for other alternatives, and their corresponding optimal $\lambda$ ). As we were interested in finding subspaces for each of the correlation matrices (which were $N=16$ in total) we computed the same number of subspaces employing one versus rest approach: for each of the main frequencies $\left(f_{i}, \mathrm{i}=1 \ldots 4\right)$ four correlation matrices related to that frequency were computed (i.e. correlations with $\sin \left(f_{i}\right), \cos \left(f_{i}\right), \sin \left(2^{*} f_{i}\right)$ and $\cos \left(2^{*} f_{i}\right)$ reference signals $)$, with dimensions number of channels by number of trials. With each of these matrices, a shrinkage LDA was trained to find the linear combination $\mathbf{w}_{N}$ best separating the classes according to Eq. 1. For each shrinkage LDA, the trials that belonged to the class with the same $f_{i}$ as the one used to calculate the corresponding correlation matrix (see Fig. 1) were taken as targets, while the remaining trials were non-targets. Thus, the final number of features per trials was reduced to $N=16$.

\subsection{Classification}

Following the procedures of [11], three classifiers were used: Decision Tree, Naïve Bayes (using kernel distribution to model the data) and K-Nearest Neighbor (KNN) (with K=5 neighbors), implemented with Statistics and Machine Learning Toolbox of Matlab.

All of them were used to calculate the training (offline) dataset classification accuracy, using a leave-one-out approach. This was done for the two techniques discussed in this paper (i.e. the standard CCA method and the new proposed pipeline, corrLDA), using three different trial lengths (1, 2 and 3 seconds).

On the contrary, the testing (online) dataset results were obtained using the best classifier for each analysed technique, estimated from the training dataset analysis. These two technique-classifier pipelines were evaluated using 1 second trial length, for the four perturbations present in the dataset, after training with the whole training dataset.

\subsection{Statistical Analysis}

We conducted a two-way analysis of variance (ANOVA) for repeated measures to analyse the effect of technique (CCA, corrLDA) and classifier (K-NN, Naïve Bayes, Decision Tree) on the results obtained with cross-validation in the training set, when using 1 second trial length. For the testing dataset, we conducted a twoway repeated measures ANOVA to see the influence of approach (the selected CCA-classifier and corrLDA-classifier combinations) and perturbation (control, listening, speaking, thinking) factors on classification results obtained with 1 second long trials.

When the interaction of factors or the main effects were significant, a Tuckey's honestly significant difference (HSD) post hoc test was computed. Furthermore, we used Mauchly's test to analyse the sphericity of the response variables in the repeated measures model of each ANOVA. When this null hypothesis was rejected, we looked at the Greenhouse-Geisser adjusted p-values. 


\section{Results}

\subsection{Training Dataset Classification}

Fig. 2 depicts mean accuracies (\%) and standard errors across subjects for each of the presented techniques. Classification results of the three tested classifiers when changing trials length from 1 to 3 seconds are represented in each of the subplots.

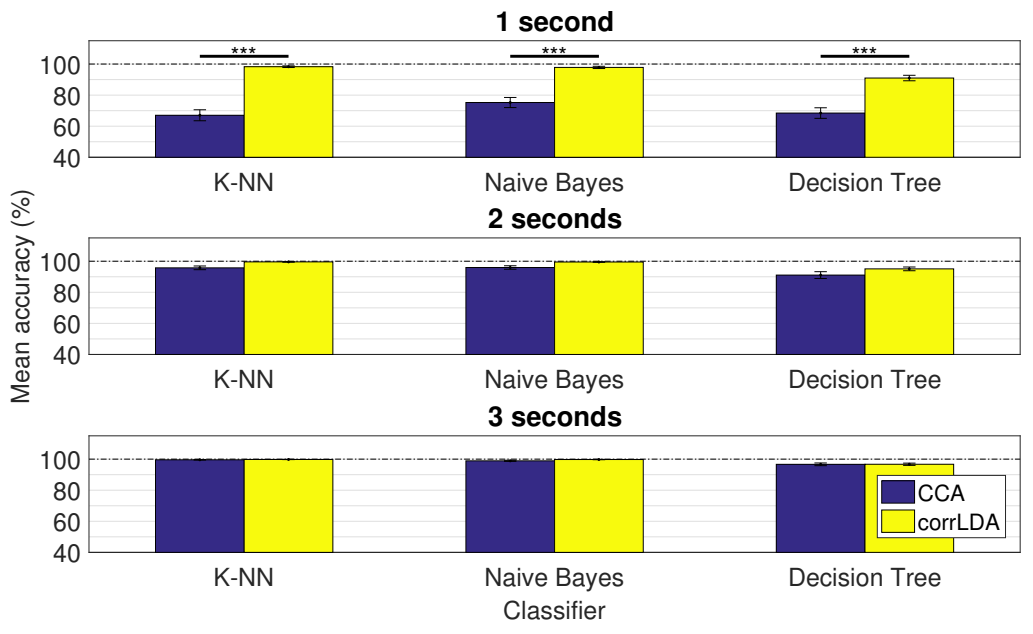

Fig. 2. Mean accuracies (\%) and standard errors for different stimuli lengths and classifiers (training dataset; 24 subjects). ${ }^{* * *}: \mathrm{p}<0.001$. Differences (Tuckey's HSD) between techniques for 1 second trial length.

As described in section 3.5, we performed a 2-way repeated measures ANOVA (see Table 1) with factors technique and classifier, using accuracy results obtained with 1 second trial length. Both main factors and their interaction were significant $(\mathrm{p}<0.001$ in each case). Thus, post-hoc tests followed.

Tuckey's HSD revealed that corrLDA outperformed CCA with the three analysed classifiers $(\mathrm{p}<0.001$ in each case). Besides, when using CCA, Naïve Bayes classifier performance was superior to K-NN and Decision Tree $(p<0.001$ in each case). On the other hand, with the corrLDA pipeline both K-NN and Naïve Bayes classifiers had better results than Decision Tree $(\mathrm{p}<0.001$ in each case).

Mean classification accuracies across subjects obtained with the proposed corrLDA pipeline were higher than $90 \%$ with 1 second long trials. This result was consistent for all three analysed classifiers (mean \pm std; K-NN: $98.29 \pm 0.53$, Naïve Bayes: $97.83 \pm 0.72$, Decision Tree: $91 \pm 1.76)$. However, for 1 second long trials, CCA could not reach $80 \%$ of mean accuracy for any of the classifiers (mean 
Table 1. Two-way repeated measures ANOVA for mean accuracies in training dataset with 1 second trial length, with classifier and technique factors.

\begin{tabular}{|l|l|l|l|l|l|}
\hline Source & $\boldsymbol{S}$ & $\boldsymbol{d}$ & $\boldsymbol{M S}$ & $\boldsymbol{F}$ & $\boldsymbol{p}$ \\
\hline Classifier & 1120.88 & 2 & 560.44 & 33.25 & $<0.001$ \\
\hline Error(Classifier) & 775.46 & 46 & 16.86 & - & - \\
\hline Technique & 23332.56 & 1 & 23332.56 & 84.28 & $<0.001$ \\
\hline Error(Technique) & 6367.27 & 23 & 276.84 & - & - \\
\hline Classifier x Technique & 603.79 & 2 & 301.90 & 15.40 & $<0.001$ \\
\hline Error(Classifier x Technique) & 901.88 & 46 & 19.61 & - & - \\
\hline
\end{tabular}

\pm std; K-NN: $67.04 \pm 3.53$, Naïve Bayes: $75.25 \pm 3.25$, Decision Tree: $68.46 \pm$ $3.40)$.

For further analyses with the testing dataset, we selected K-NN and Naïve Bayes classifiers to analyse corrLDA and CCA techniques, respectively (i.e. corrLDA-KNN and CCA-NB).

\subsection{Testing Dataset Classification}

As previously mentioned, two technique-classifier approaches were evaluated with the testing dataset: CCA-NB and corrLDA-KNN. In Fig. 3 mean accuracies (\%) and standard errors across subjects are shown for each studied approach under different perturbations (using 1 second long trials).

A two-way ANOVA for repeated measures was conducted (see Table 2) with approach and perturbation factors. The results showed that both main factors were significant $(\mathrm{p}<0.001$ in each case), but there was no significant interaction between them $(\mathrm{p}=0.4435)$.

Table 2. Two-way repeated measures ANOVA for mean accuracies in testing dataset with 1 second trial length, with approach and perturbation factors.

\begin{tabular}{|l|l|l|l|l|l|}
\hline Source & $\boldsymbol{S}$ & $\boldsymbol{d f}$ & $\boldsymbol{M S}$ & $\boldsymbol{F}$ & $\boldsymbol{p}$ \\
\hline Approach & 41477.52 & 1 & 41477.52 & 225.21 & $<0.001$ \\
\hline Error(Approach) & 4235.98 & 23 & 184.17 & - & - \\
\hline Perturbation & 2526.71 & 3 & 842.24 & 13.38 & $<0.001$ \\
\hline Error(Perturbation) & 4342.29 & 69 & 62.93 & - & - \\
\hline Approach x Perturbation & 80.44 & 3 & 26.81 & 0.84 & 0.4435 \\
\hline Error(Approach x Perturbation) & 2211.06 & 69 & 32.04 & - & - \\
\hline
\end{tabular}

Both approaches got the highest mean accuracy result in Control condition (mean \pm std; corrLDA-KNN: $88.08 \pm 1.73$, CCA-NB: $59.00 \pm 2.41$ ), followed by Listening (mean \pm std; corrLDA-KNN: $85.58 \pm 2.24$, CCA-NB: $56.04 \pm 2.30$ ), Thinking (mean \pm std; corrLDA-KNN: $82.58 \pm 2.45$, CCA-NB: $51.29 \pm 2.40$ ) and Speaking (mean \pm std; corrLDA-KNN: $77.88 \pm 3.38$, CCA-NB: $50.21 \pm$ 2.33) perturbations. 


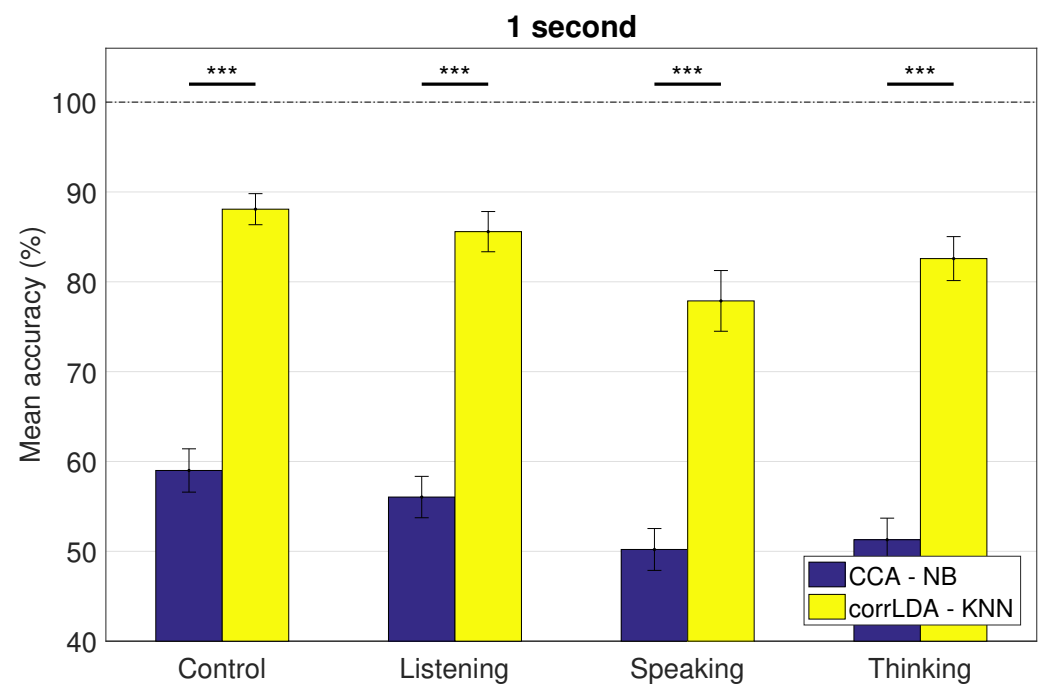

Fig. 3. Mean accuracies (\%) and standard errors for different perturbations with 1 second trial length (testing dataset; 24 subjects). ${ }^{* * *}: \mathrm{p}<0.001$. Differences (Tuckey's HSD) between approaches for each perturbation.

Finally, post-hoc tests revealed that the CCA-NB approach achieved significantly less accuracy than corrLDA-KNN for all perturbations $(\mathrm{p}<0.001$, in all cases).

\section{Discussion}

As observed from previous results, the corrLDA pipeline outperforms CCA. CCA-based methods do not take into account between-class information to compute correlations, because each CCA is performed with reference signals of just one class. Therefore, when features from different classes overlap, which on the other hand is the most common setting for SSVEP signals [20], the classification results are poor. On the contrary, the corrLDA pipeline employs linear discriminant functions that maximize the difference between classes, thus (linearly) reducing the overlap between them. Consequently, and as we have seen, corrLDA achieves significantly better performance than CCA.

Furthermore, corrLDA achieves higher mean accuracy classification results with short trial lengths than CCA. In particular, we analysed trials of 1 second length, with which it was possible to achieve almost $90 \%$ mean accuracy in the control condition (without noise perturbations). In the case of CCA, however, the mean accuracy was around $60 \%$ for condition control. The reason for this is that regression-based methods are unable to achieve reliable estimates without enough number of cycles of the signal of interest. It is clear thus, that our pro- 
posed pipeline corrLDA can be considered a good option to design fast and easy to train SSVEP-based BCI systems, with the potential of increasing information transfer rates [41].

Regarding classification results achieved under different perturbations, our corrLDA pipeline again outperforms the classification results obtained with CCA, in the four analysed conditions. Even without including knowledge of the noise structure of each perturbation, corrLDA achieved an average of $83.53 \%$ of accuracy under all analysed disturbances, whereas CCA only reached $54.14 \%$. Nevertheless, both techniques are affected by noise, being the speaking condition the one that most affects (decreases) mean accuracies. As stated in [11], the reason for this could be that the quality of the EEG signal decreases in the theta band. Another reason could be the attention split that the subjects undergo due to dual tasking. Indeed, the latter factor is also present in the thinking condition, which is the next worst condition regarding classification performance. These results suggest that further investigation needs to be done in order to develop BCI systems that are robust against perturbations, so that they can be reliably used in out-of-the-lab environments.

Finally, the proposed pipeline is practical and easy to use since it does not require to select subject-specific hyper-parameters, which greatly simplifies the set-up of the system. Unlike CCA, corrLDA needs some calibration data in order to train the shrink LDAs. corrLDA offers, however, significantly better performance than CCA, even under noisy conditions and using significantly shorter trial lengths.

\section{Conclusions}

In this paper we proposed a new pipeline, named corrLDA, to develop a fast SSVEP-based BCI system. This pipeline computes correlation values with sinecosine reference templates and uses shrinkage LDA to reduce the number of features. Finally, a non-linear classifier is used to decode the EEG signal. We showed that corrLDA outperforms CCA using trial lengths as short as 1 second, under both ideal and noisy conditions. Further investigations need to be done to robustly classify SSVEP data regardless of the perturbation.

Acknowledgements We are grateful to Zafer İşcan for partly sharing code and to Mina Jamshidi and Vadim V. Nikulin for fruitful discussions.

\section{References}

1. Ahn, M., Lee, M., Choi, J., Jun, S.: A review of brain-computer interface games and an opinion survey from researchers, developers and users. Sensors 14(8), 1460114633 (2014). https://doi.org/10.3390/s140814601

2. Bin, G., Gao, X., Yan, Z., Hong, B., Gao, S.: An online multi-channel ssvepbased brain-computer interface using a canonical correlation analysis method. Journal of neural engineering 6(4), 046002 (2009). https://doi.org/10.1088/17412560/6/4/046002 
3. Blankertz, B., Lemm, S., Treder, M., Haufe, S., Müller, K.R.: Single-trial analysis and classification of erp components - a tutorial. NeuroImage 56(2), 814-825 (2011). https://doi.org/10.1016/j.neuroimage.2010.06.048

4. Blankertz, B., Tangermann, M., Vidaurre, C., Fazli, S., Sannelli, C., Haufe, S., Maeder, C., Ramsey, L.E., Sturm, I., Curio, G., et al.: The berlin brain-computer interface: non-medical uses of bci technology. Frontiers in neuroscience 4, 198 (2010). https://doi.org/10.3389/fnins.2010.00198

5. Cao, L., Ju, Z., Li, J., Jian, R., Jiang, C.: Sequence detection analysis based on canonical correlation for steady-state visual evoked potential brain computer interfaces. Journal of neuroscience methods 253, 10-17 (2015). https://doi.org/10.1016/j.jneumeth.2015.05.014

6. Daly, J.J., Wolpaw, J.R.: Brain-computer interfaces in neurological rehabilitation. The Lancet Neurology 7(11), 1032-1043 (2008). https://doi.org/10.1016/S14744422(08)70223-0

7. Dobkin, B.H.: Brain-computer interface technology as a tool to augment plasticity and outcomes for neurological rehabilitation. The Journal of physiology 579(3), 637-642 (2007). https://doi.org/10.1113/jphysiol.2006.123067

8. Farooq, M., Dehzangi, O.: High accuracy wearable ssvep detection using feature profiling and dimensionality reduction. In: 2017 IEEE 14th International Conference on Wearable and Implantable Body Sensor Networks (BSN). pp. 161-164. IEEE (2017). https://doi.org/10.1109/BSN.2017.7936032

9. Friman, O., Volosyak, I., Graser, A.: Multiple channel detection of steady-state visual evoked potentials for brain-computer interfaces. IEEE transactions on biomedical engineering 54(4), 742-750 (2007). https://doi.org/10.1109/TBME.2006.889160

10. Gao, X., Xu, D., Cheng, M., Gao, S.: A bci-based environmental controller for the motion-disabled. IEEE Transactions on neural systems and rehabilitation engineering 11(2), 137-140 (2003). https://doi.org/10.1109/TNSRE.2003.814449

11. Işcan, Z., Nikulin, V.V.: Steady state visual evoked potential (ssvep) based braincomputer interface (bci) performance under different perturbations. PloS one 13(1), e0191673 (2018). https://doi.org/10.1371/journal.pone.0191673

12. Kawanabe, M., Vidaurre, C., Blankertz, B., KR, M.: A maxmin approach to optimize spatial filters for eeg single-trial classification. In: International Work-Conference on Artificial Neural Networks. pp. 674-682. Springer (2009). https://doi.org/10.1007/978-3-642-02478-8_84

13. Kübler, A., Furdea, A., Halder, S., Hammer, E.M., Nijboer, F., Kotchoubey, B.: A brain-computer interface controlled auditory event-related potential (p300) spelling system for locked-in patients. Annals of the New York Academy of Sciences 1157(1), 90-100 (2009). https://doi.org/10.1111/j.1749-6632.2008.04122.x

14. Kübler, A., Nijboer, F., Mellinger, J., Vaughan, T.M., Pawelzik, H., Schalk, G., McFarland, D.J., Birbaumer, N., Wolpaw, J.R.: Patients with als can use sensorimotor rhythms to operate a brain-computer interface. Neurology 64(10), 17751777 (2005). https://doi.org/10.1212/01.WNL.0000158616.43002.6D

15. Ledoit, O., Wolf, M.: Honey, i shrunk the sample covariance matrix. The Journal of Portfolio Management 30(4), 110-119 (2004). https://doi.org/10.3905/jpm.2004.110

16. Ledoit, O., Wolf, M., et al.: A well-conditioned estimator for large-dimensional covariance matrices. Journal of Multivariate Analysis 88(2), 365-411 (2004)

17. Lin, Z., Zhang, C., Wu, W., Gao, X.: Frequency recognition based on canonical correlation analysis for ssvep-based bcis. IEEE transactions on biomedical engineering 53(12), 2610-2614 (2006). https://doi.org/10.1109/TBME.2006.886577 
18. Lorenz, R., Pascual, J., Blankertz, B., Vidaurre, C.: Towards a holistic assessment of the user experience with hybrid bcis. Journal of neural engineering 11(3), 035007 (2014)

19. McFarland, D.J., Wolpaw, J.R.: Brain-computer interface operation of robotic and prosthetic devices. Computer 41(10), 52-56 (2008). https://doi.org/10.1109/MC.2008.409

20. Nakanishi, M., Wang, Y., Chen, X., Wang, Y.T., Gao, X., Jung, T.P.: Enhancing detection of ssveps for a high-speed brain speller using task-related component analysis. IEEE Transactions on Biomedical Engineering 65(1), 104-112 (2017). https://doi.org/10.1109/TBME.2017.2694818

21. Nan, W., Wong, C.M., Wang, B., Wan, F., Mak, P.U., Mak, P.I., Vai, M.I.: A comparison of minimum energy combination and canonical correlation analysis for ssvep detection. In: 2011 th International IEEE/EMBS Conference on Neural Engineering. pp. 469-472. IEEE (2011). https://doi.org/10.1109/NER.2011.5910588

22. Nierhaus, T., Vidaurre, C., Sannelli, C., Mueller, K.R., Villringer, A.: Immediate brain plasticity after one hour of brain-computer interface (bci). The Journal of physiology (2019). https://doi.org/10.1113/JP278118

23. Nijholt, A., Bos, D.P.O., Reuderink, B.: Turning shortcomings into challenges: Brain-computer interfaces for games. Entertainment computing 1(2), 85-94 (2009). https://doi.org/10.1016/j.entcom.2009.09.007

24. Perez, J.L.M., Cruz, A.B.: Linear discriminant analysis on brain computer interface. In: 2007 IEEE International Symposium on Intelligent Signal Processing. pp. 1-6. IEEE (2007). https://doi.org/10.1109/WISP.2007.4447590

25. Pfurtscheller, G., Brunner, C., Schlögl, A., Da Silva, F.L.: Mu rhythm (de) synchronization and eeg single-trial classification of different motor imagery tasks. NeuroImage 31(1), 153-159 (2006). https://doi.org/10.1016/j.neuroimage.2005.12.003

26. Pfurtscheller, G., Flotzinger, D., Kalcher, J.: Brain-computer interface - a new communication device for handicapped persons. Journal of Microcomputer Applications 16(3), 293-299 (1993). https://doi.org/10.1006/jmca.1993.1030

27. Saa, J.F.D., Gutierrez, M.S.: Eeg signal classification using power spectral features and linear discriminant analysis: A brain computer interface application. In: Eighth Latin American and Caribbean Conference for Engineering and Technology. pp. 17. LACCEI Arequipa (2010)

28. Sannelli, C., Vidaurre, C., Müller, K.R., Blankertz, B.: A large scale screening study with a smr-based bci: Categorization of bci users and differences in their smr activity. PloS one 14(1) (2019). https://doi.org/10.1371/journal.pone.0207351

29. Schäfer, J., Strimmer, K.: A shrinkage approach to large-scale covariance matrix estimation and implications for functional genomics. Statistical applications in genetics and molecular biology 4(1) (2005). https://doi.org/10.2202/1544-6115.1175

30. Sellers, E.W., Krusienski, D.J., McFarland, D.J., Vaughan, T.M., Wolpaw, J.R.: A p300 event-related potential brain-computer interface (bci): the effects of matrix size and inter stimulus interval on performance. Biological psychology 73(3), 242252 (2006). https://doi.org/10.1016/j.biopsycho.2006.04.007

31. Srinivasan, R., Bibi, F.A., Nunez, P.L.: Steady-state visual evoked potentials: distributed local sources and wave-like dynamics are sensitive to flicker frequency. Brain topography 18(3), 167-187 (2006). https://doi.org/10.1007/s10548006-0267-4

32. Vecchiato, G., Astolfi, L., Fallani, F.D.V., Salinari, S., Cincotti, F., Aloise, F., Mattia, D., Marciani, M.G., Bianchi, L., Soranzo, R., et al.: The study of brain activity during the observation of commercial advertsing by using 
high resolution eeg techniques. In: 2009 Annual International Conference of the IEEE Engineering in Medicine and Biology Society. pp. 57-60. IEEE (2009). https://doi.org/10.1109/IEMBS.2009.5335045

33. Vidaurre, C., Klauer, C., Schauer, T., Ramos-Murguialday, A., Mueller, K.R.: Eeg-based bci for the linear control of an upper-limb neuroprosthesis. Medical engineering \& physics 38, 1195-1204 (2016). https://doi.org/10.1016/j.medengphy.2016.06.010

34. Vidaurre, C., Murguialday, A.R., Haufe, S., Gómez, M., Müller, K.R., Nikulin, V.V.: Enhancing sensorimotor bci performance with assistive afferent activity: An online evaluation. NeuroImage 199, 375-386 (2019). https://doi.org/10.1016/j.neuroimage.2019.05.074

35. Vidaurre, C., Pascual, J., Ramos-Murguialday, A., Lorenz, R., Blankertz, B., Birbaumer, N., Müller, K.R.: Neuromuscular electrical stimulation induced brain patterns to decode motor imagery. Clinical Neurophysiology 124(9), 1824-1834 (2013). https://doi.org/10.1016/j.clinph.2013.03.009

36. Vidaurre, C., Sannelli, C., Müller, K., Blankertz, B.: Machine-learning based coadaptive calibration: a perspective to fight bci illiteracy. In: International Conference on Hybrid Artificial Intelligence Systems. pp. 413-420. Springer (2010). https://doi.org/10.1007/978-3-642-13769-3_50

37. Vidaurre, C., Krämer, N., Blankertz, B., Schlögl, A.: Time domain parameters as a feature for eeg-based brain-computer interfaces. Neural Networks 22(9), 13131319 (2009). https://doi.org/10.1016/j.neunet.2009.07.020

38. Vidaurre, C., Scherer, R., Cabeza, R., Schlögl, A., Pfurtscheller, G.: Study of discriminant analysis applied to motor imagery bipolar data. Medical \& biological engineering \& computing 45(1), 61 (2007). https://doi.org/10.1007/s11517-0060122-5

39. Wang, Y., Gao, X., Hong, B., Jia, C., Gao, S.: Brain-computer interfaces based on visual evoked potentials. IEEE Engineering in medicine and biology magazine 27(5), 64-71 (2008). https://doi.org/10.1109/MEMB.2008.923958

40. Wang, Y., Wang, R., Gao, X., Hong, B., Gao, S.: A practical vep-based braincomputer interface. IEEE Transactions on neural systems and rehabilitation engineering 14(2), 234-240 (2006). https://doi.org/10.1109/TNSRE.2006.875576

41. Wolpaw, J.R., Birbaumer, N., McFarland, D.J., Pfurtscheller, G., Vaughan, T.M.: Brain-computer interfaces for communication and control. Clinical neurophysiology 113(6), 767-791 (2002). https://doi.org/10.1016/S1388-2457(02)00057-3

42. Yin, E., Zhou, Z., Jiang, J., Yu, Y., Hu, D.: A dynamically optimized ssvep braincomputer interface (bci) speller. IEEE Transactions on Biomedical Engineering 62(6), 1447-1456 (2014). https://doi.org/10.1109/TBME.2014.2320948

43. Zhang, R., Xu, P., Guo, L., Zhang, Y., Li, P., Yao, D.: Z-score linear discriminant analysis for eeg based brain-computer interfaces. PloS one 8(9) (2013). https://doi.org/10.1371/journal.pone.0074433

44. Zhang, Y., Zhou, G., Jin, J., Wang, M., Wang, X., Cichocki, A.: L1-regularized multiway canonical correlation analysis for ssvep-based bci. IEEE transactions on neural systems and rehabilitation engineering 21(6), 887-896 (2013). https://doi.org/10.1109/TNSRE.2013.2279680

45. Zhang, Y., Zhou, G., Jin, J., Wang, X., Cichocki, A.: Frequency recognition in ssvep-based bci using multiset canonical correlation analysis. International journal of neural systems 24(04), 1450013 (2014). https://doi.org/10.1142/S0129065714500130 